\title{
Vibrating wires for beam diagnostics
}

\author{
S.G. Arutunian ${ }^{\mathrm{a}, *}$, M.R. Mailian, ${ }^{\mathrm{a}}$, Kay Wittenburg ${ }^{\mathrm{b}}$ \\ ${ }^{a}$ Yerevan Physics Institute, Alikhanian Br. Str. 2, 375036 Yerevan, Armenia \\ ${ }^{\mathrm{b}} \mathrm{DESY}$, Germany
}

Received 11 August 2005; received in revised form 19 December 2006; accepted 27 December 2006

Available online 10 January 2007

\begin{abstract}
A new approach to the technique of particle beam scanning by wires has been developed. The novelty of the method is that the wire heating quantity is used as a source of information about the number of interacting particles. The wire heating measurement is performed as a change of the wire natural oscillation frequency. The excitation of wire natural oscillations is provided by interaction of a current through the wire with a permanent magnetic field. A shift in the wire natural oscillation frequency characterizes the change in the conditions of wire irradiation by the measured beam. By the rigid fixing of the wire ends on the support an unprecedented sensitivity of the frequency to the temperature and to the corresponding flux of colliding particles is obtained. The range of frequencies used (about $10 \mathrm{kHz}$ ) and characteristic time of heat transfer limit the speed characteristics of the proposed scanning method; however, the high sensitivity makes it a prospective one for investigation of beam halo and weak beam scanning. Traditional beam profile monitors generally focus on the beam core and loose sensitivity in the halo region where a large dynamic range of detection is necessary. The scanning by a vibrating wire can also be used in profiling and detecting of neutron and photon beams.
\end{abstract}

(C) 2007 Elsevier B.V. All rights reserved.

PACS: 29.27.Fh

Keywords: Accelerator; Diagnostics; Beam; Profiling; Vibrating; Wire

\section{Introduction}

One of the widespread methods of beam profiling in accelerators is the scanning of charged particles beams by thin wires (see e.g., Refs. [1-4]). The principle of operation is based on the measurement of fluxes of secondary particles scattered off the wire, the intensity of which is proportional to the quantity of primary particles colliding with the wire.

In this paper a new approach to the technique of scanning by wires is developed. The novelty of the method is that the wire heating quantity is used as a source of information about the number of interacting particles. The wire heating measurement is performed as a change of wire natural oscillations frequency. The excitation of wire natural oscillations is provided by interaction of a current through the wire with a permanent magnetic field.

\footnotetext{
${ }^{*}$ Corresponding author. Tel./fax: + 3741350114 .

E-mail address: femto@yerphi.am (S.G. Arutunian).
}

A shift in the wire natural oscillation frequency characterizes the change in the conditions of wire irradiation by the measured beam. By the rigid fixing of the wire ends on the support an unprecedented sensitivity of the frequency to the temperature and to the corresponding flux of colliding particles is obtained. The idea to use the vibrating wire frequency for beam diagnostics was suggested in Refs. [5,6]. Such vibrating wire scanners (VWS) were used for electron, ion, proton and photon beams profiling.

The range of used frequencies (about $10 \mathrm{kHz}$ ) and characteristic time of processes of heat transfer limit the speed characteristics of proposed scanning method, however, the high sensitivity makes it a prospective one for investigation of beam halo and weak beam scanning [7]. Traditional beam profile monitors generally focus on the beam core and loose sensitivity in the halo region where a large dynamic range of detection is necessary.

The scanning by a vibrating wire can be also used in profiling and detecting of neutron and photon beams. 


\section{Description of the method}

The first techniques based on the vibrating wire technologies were developed in the late 1920s and early 1930s [8]. Today the area of application of this technique expanded and the number of vibrating wire-based instruments has increased. The operating principle is based on the measurement of the change in the frequency of a vibrating wire, which is stretched on a support, depending on the physical parameters of the wire and environment in which oscillations take place.

The important advantages of properly constructed vibrating wire sensors are inherent long-term stability, high precision and resolution, good reproducibility and small hysteresis. The advantage of vibrating wire sensors is also that the frequency signal is imperturbable and can be transmitted over long cable with no loss or degradation of the signal. The reliability of the sensors becomes the overriding feature in the selection of a technology. It is also important to ascertain a no-zero drift and minimum change in sensitivity. An important parameter of vibrating wire-based sensors is their capability to operate in hard conditions (high operational, power and temperature cycling, thermal shock, thermal storage, autoclave, fluid immersion, mechanical shock, electromagnetic irradiation, electrostatic [9]).

We take an electromechanical resonator with a metallic vibrating wire excited by the interaction of a current with a permanent magnetic field as a base for the VWS of electron and proton beams. In this paper we discuss an application of such resonators for precise measurement of profile of low current particles beams. The interaction of the beam with the wire mainly causes heating of the wire. Thus, we expect that the frequency of natural oscillations of the wire will provide information about its temperature. Given that much more energy is deposited in heat than in secondary particles (for instance, it is estimated that $35.5 \mathrm{keV}$ of the $100 \mathrm{MeV}$ proton beam energy is deposited for heating the wire, and only $0.67 \mathrm{keV}$ of energy is transformed into secondary radiation or particles [1]) we expect that using the wire temperature to measure the beam profile will be much more efficient than traditional techniques.

The thermal method of measurement also allows registering fluxes of neutrons and photons.

\subsection{Wire oscillations frequency temperature dependence}

A vibrating wire-based electromechanical resonator roughly can be represented as a support with a strained vibrating wire.

The frequency of the first harmonics of the wire oscillations can be written in the form [10]

$f=\frac{1}{2 l} \sqrt{\sigma / \rho}\left(1+\frac{d}{2 l} \sqrt{E / \sigma}\right)$.

Here $\sigma$ is the stress of the wire with length $l$ and diameter $d$, $\rho$ and $E$ are the density of wire material and modulus of elasticity. The second term in formula (1) corresponds to the wire elasticity. In formula (1) it is supposed that the wire is sufficiently thin and strained; therefore, the wire stress is much more than $(d / 2 l)^{2} E$.

If the resonator is heated, the oscillation frequency of a vibrating wire has a strong temperature dependence due to temperature stress. Let the initial stress of the wire be $\sigma_{0}=E\left(l_{\mathrm{B} 0}-l_{\mathrm{S} 0}\right) / l_{\mathrm{S} 0}$, where length of unstrained wire was $l_{\mathrm{S} 0}$ and the distance between points of wire ends fixation was $l_{\mathrm{B} 0}$ (resonator was assembled at initial temperature $T_{0}$ ). The rigidity of the support is much more than that of the wire hence the wire length is defined by the distance between clips of the support. In the general case of electromechanical resonator operation we can suppose that the wire temperature has an average value $T_{\mathrm{S}}$ along the wire, and the average temperature of the base is $T_{\mathrm{B}}$. The relative change of the wire frequency is

$$
\begin{aligned}
\Delta f / f & =0.5 \cdot \Delta \sigma / \sigma_{0} \\
& =0.5 \cdot\left(E / \sigma_{0}\right)\left(\alpha_{\mathrm{B}}\left(T_{\mathrm{B}}-T_{0}\right)-\alpha_{\mathrm{S}}\left(T_{\mathrm{S}}-T_{0}\right)\right)
\end{aligned}
$$

here $\alpha_{B}$ and $\alpha_{S}$ are the coefficients of thermal expansion of the support and wire.

The wire initial stress must be less of the elasticity limit, otherwise the sensor's frequency will have drifts. In developed pickups the support is maintained at room temperature and the information source is the wire temperature. The less the wire strain at assembly, the less is the temperature increment at which the wire will be loosened and broken due to oscillations. To estimate the temperature maximal increment take the yield stress $\sigma_{0.2}$ (stress, for which the residual deformation after removal of the load is $0.2 \%$ ) as the maximal stress. Also the maximum of wire overheating temperature is defined as $\Delta T_{\mathrm{S}}^{\mathrm{MAX}}=\sigma_{0.2} / \alpha_{\mathrm{S}} E$. In Table 1 characteristic values for different materials are presented $[11,12]$.

In the data marked by a "*" sign the half of the breaking load is taken as $\sigma_{0.2}$. From the table it is seen that Titan alloys and $\mathrm{SiC}$ threads hold sufficiently a large thermal range.

\subsection{Wire under beam irradiation}

The interaction of the beam with the wire mainly causes heating of the wire due to the energy loss of the particles in the wire. For charged particles the main parameter of energy transfer is the particle ionization losses $\mathrm{d} E / \mathrm{d} s$.

The average heating quantity $q_{1}$ from one particle passing though the wire is

$q_{1}=K_{\mathrm{tr}} \frac{\mathrm{d} E}{\mathrm{~d} s} \frac{\pi}{2} r$

where $K_{\text {tr }}$ describes the transformation ratio of ionization losses into heat (we suppose $K_{\mathrm{tr}}=0.3$ ) and the wire radius is $r, \mathrm{~d} E / \mathrm{d} s$ is ionization losses of particles in wire material. The value $(\pi / 2) r$ describes the mean path of particles passing the wire. We suppose that the beam has a Gaussian current distribution with amplitude $I_{0}$ and horizontal size 
$\sigma_{x}$. We choose the following coordinate system: axis $x$-in the horizontal plane and orthogonal to the beam direction directed to outside of accelerator ring, axis $z$-vertical direction, $s$-beam propagation direction. The current of particles $I_{\mathrm{S}}$ deposited on the wire is defined by the expression

$I_{\mathrm{S}}=I_{0} \cdot \frac{2 r}{\sigma_{x} \sqrt{2 \pi}} \exp \left(-\frac{x^{2}}{2 \sigma_{x}^{2}}\right)$

where $x$ is the distance between the wire and the beam center.

The power $Q_{\mathrm{S}}$ heating the wire is: $Q_{\mathrm{S}}=q_{1} I_{\mathrm{S}} / e(e-$ electron charge). The main cooling mechanism at room temperature is thermoconductivity. Therefore, $Q_{\mathrm{S}}$ causes a wire heating with respect to the environment temperature

Table 1

Temperature range of vibrating wire sensor

\begin{tabular}{llllc}
\hline Material & $E(\mathrm{GPa})$ & $\alpha_{\mathrm{S}}(1 / \mathrm{K})$ & $\sigma_{0.2}(\mathrm{GPa})$ & $\Delta T_{\mathrm{S}}^{\mathrm{MAX}}(\mathrm{K})$ \\
\hline $\begin{array}{l}\text { Beryl Bronze } \\
\text { hard }\end{array}$ & 130 & $1.90 \mathrm{E}-5$ & $0.9^{*}$ & 482 \\
$\begin{array}{l}\text { Tungsten } \\
\text { Titan }\left(\sigma_{0.2} \text { at }\right.\end{array}$ & 400 & $4.70 \mathrm{E}-6$ & $0.5^{*}$ & 433 \\
$\left.20^{\circ} \mathrm{C}\right)$ & 110 & $9.86 \mathrm{E}-6$ & 0.66 & 909 \\
$\begin{array}{l}\text { Titan, thermally } \\
\text { treated, } 99.6 \%\end{array}$ & 110 & $9.86 \mathrm{E}-6$ & 0.3 & 577 \\
$\begin{array}{l}\text { Platinum, } \\
\text { thermally }\end{array}$ & 160 & $9.70 \mathrm{E}-6$ & 0.07 & 341 \\
$\begin{array}{l}\text { treated } \\
\text { SiC, fiber }\end{array}$ & & & & \\
SiC (whiskers) & 400 & $4.50 \mathrm{E}-6$ & $2^{*}$ & 856 \\
$\mathrm{SiO}_{2}$ fiber & 550 & $4.60 \mathrm{E}-6$ & $3.05^{*}$ & 1770 \\
& & $6.25 \mathrm{E}-6$ & $1.38^{*}$ & 702
\end{tabular}

The maximal temperature shift $\Delta T_{\mathrm{S}}$ of a wire with coefficient of thermal expansion $\alpha_{\mathrm{S}}$, and elasticity modulus $E$ is defined for preliminary stress of the wire equal to yield stress $\sigma_{0.2}$. by the value

$$
T_{\text {mean }}=g_{\mathrm{F}} \frac{Q_{\mathrm{S}} l}{8 \pi r^{2} \lambda}
$$

where $\lambda$ is the coefficient of thermal conductivity of the wire, form-factor $g_{\mathrm{F}} \approx 1$ determines the difference between this simple model and exact solution of thermoconductivity.

The formula for a frequency shift due to the wire heating by a mean temperature $T_{\text {mean }}$ is given by

$\Delta f=-0.25(f E / \sigma) \alpha_{\mathrm{S}} T_{\text {mean }}$.

\subsection{Dynamics of the wire heating}

Dynamic characteristics of the wire heating process are needed to choose the VWS scanning speed. To define this parameter, the heat conductivity dynamic equation was solved numerically. In a vacuum chamber of an accelerator the wire's thermal balance is determined by a heat deposit in the wire material caused by beam particles, heat transfer caused by thermal conductivity along the wire and radiation of heat from the wire's surface.

The transverse density of the beam current is modeled by a Gaussian distribution. We also considered temperature dependencies of the heat capacity and the heat conductivity of the wire.

Fig. 1 presents the temperature profiles along the wire for different times after a wire irradiation by a $15 \mathrm{GeV}$ proton beam with a mean beam current $1 \mathrm{~mA}$. It was taken into account that the wire periphery is partly covered by the magnet system. The beam width $\sigma_{x}$ in horizontal plane was set to $6 \mathrm{~mm}$. One can see that the temperature distribution trends to a triangle. The parameters of the wire for the calculation above were: material-Beryl-Bronze, diameter $-90 \mu \mathrm{m}$, length $-36 \mathrm{~mm}$. Wire placement $-20 \mathrm{~mm}$

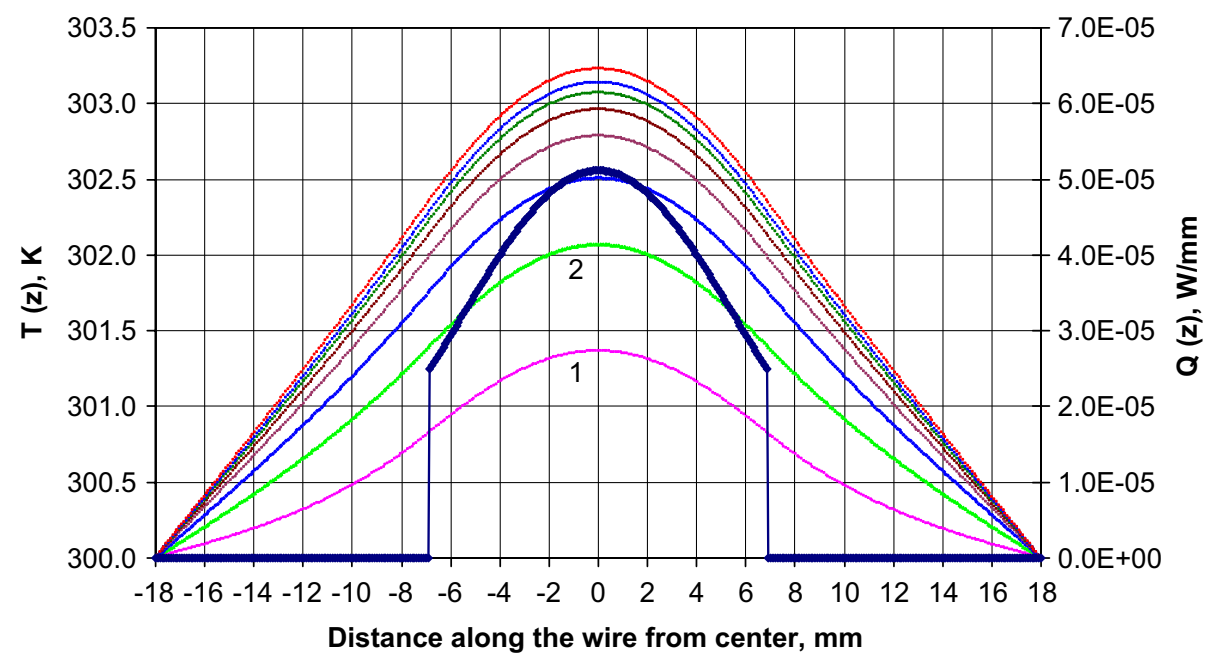

Fig. 1. Irradiated wire temperature profile calculations at different times. As a sample of a vibrating wire a Beryl-Bronze wire with diameter of $90 \mu \mathrm{m}$ and length of $36 \mathrm{~mm}$ is chosen. The corresponding power density $Q(z)$ along the wire length is presented by the bold curve. Only distances of -7 to $+7 \mathrm{~mm}$ from the center of the VWM are open for interaction with the proton beam. The numbers denote: 1 - temperature profile $T(z)$ after $1 \mathrm{~s}$ of wire irradiation start, 2 - after $2 \mathrm{~s}$ etc. till $7 \mathrm{~s}$ and then for $10 \mathrm{~s}$. 
from beam center in vertical direction. The bold line represents the linear distribution of the power deposited on the wire. Numbers 1 and 2 show the dynamic temperature profiles 1 and $2 \mathrm{~s}$ after the beam hits the wire.

The dynamics of the wire maximal temperature and corresponding frequency shifts are presented in Fig. 2. One can observe that the maximal shift of frequency trends to approximately $40 \mathrm{~Hz}$. The data obtained are used for optimizing the scanner feed.

Temperature profiles of wires from different materials are calculated to choose the optimum wire material. the geometrical parameters were always the same: the diameter was $90 \mu \mathrm{m}$, the length was $36 \mathrm{~mm}$. In these calculations the wire heating process is modeled by a $15 \mathrm{GeV} 1 \mathrm{~mA}$ proton beam with a width of $\sigma_{x}=6 \mathrm{~mm}$. The wire is placed at $3.3 \sigma_{x}$ from the beam center. Fig. 3 presents the results of such calculations for Titan, Beryl-Bronze, Platinum and Tungsten.

\subsection{Choice of VWS parameters}

As a good place to install a scanning experiment we consider to use the proton beam of the PETRA accelerator at the Deutsches Elektronen Synchrotron, DESY, Hamburg, Germany. Typical values of the proton beam in the long-time operation mode are beam energy of $15 \mathrm{GeV}$ and beam current of about $10 \mathrm{~mA}$.

Wire diameter and material: We first choose the wire diameter because this parameter determines the spatial resolution of the scanning. Characteristic sizes of the PETRA accelerator proton beam are 5 to $6 \mathrm{~mm}$ both in horizontal and vertical directions. For such a beam,

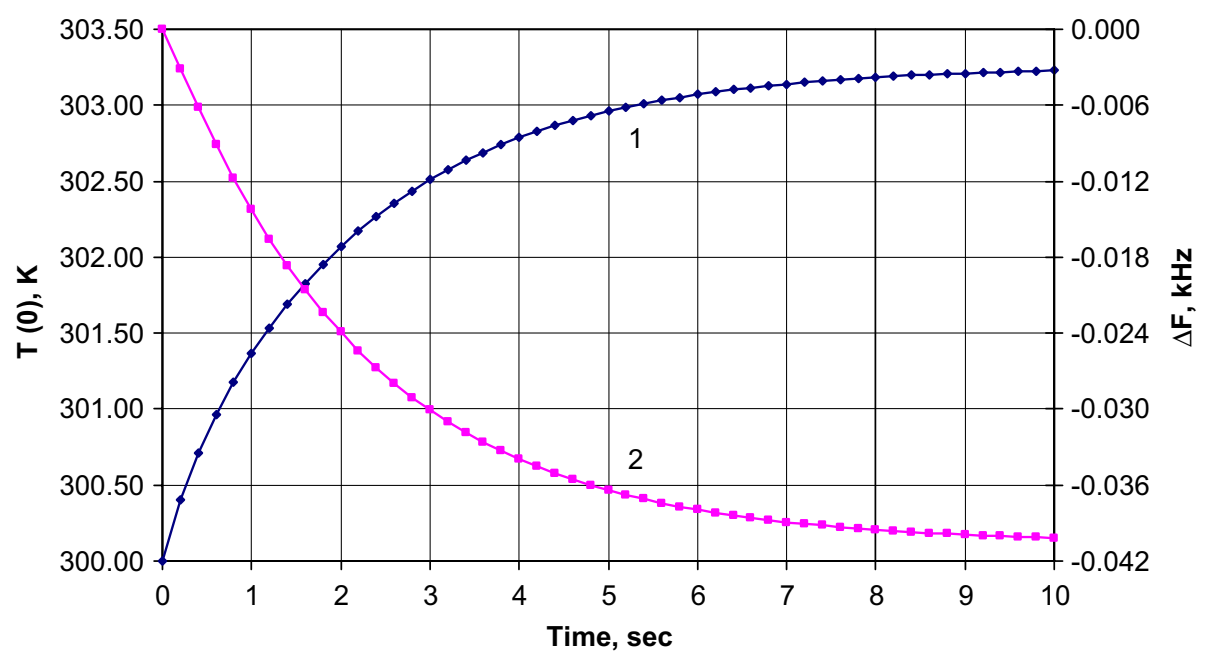

Fig. 2. Dynamics of temperature of the wire's central point - 1 ; wire natural frequency (second harmonic) - 2. In the figure are presented the numerical results of calculations for a vibrating wire from Beryl-Bronze of diameter of $90 \mu \mathrm{m}$ and length of $36 \mathrm{~mm}$ (see Fig. 1). The initial frequency of the wire is $4850 \mathrm{~Hz}$.

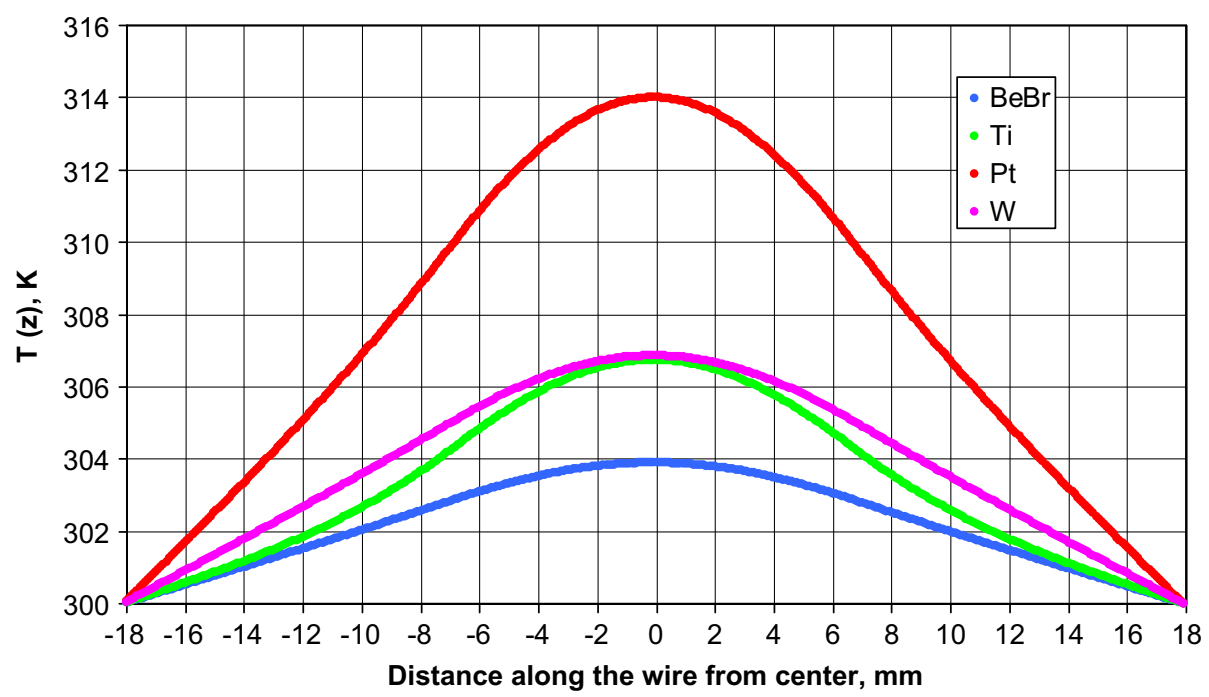

Fig. 3. Temperature profiles in the wires from Beryl-Bronze, Titan, Platinum and Tungsten irradiated by $1 \mathrm{~mA}$ and $15 \mathrm{GeV}$ proton beam $\left(\sigma_{x}=6 \mathrm{~mm}\right)$. The geometrical characteristics of the wires are the same- diameter of $90 \mu \mathrm{m}$ and length of $36 \mathrm{~mm}$. 
a scanning with an accuracy of fractions of a millimeter is adequate. A diameter of the wire of $90 \mu \mathrm{m}$ is convenient for assembling and is adequate also for the required spatial resolution. Since the experiment is aimed to scan the beam periphery only, it is important to select the wire material with sufficiently large coefficient of thermal expansion, to achieve a high sensitivity of the pickup. Stable mechanical characteristics of the wire are very important as well. BerylBronze satisfies both criteria. The maximal operational temperature range at wire stresses near the linear limit of 20 to $140^{\circ} \mathrm{C}$ is also suitable.

Wire length: Our experience with vibrating wire-based electromechanical resonators showed that stable characteristics of the resonator are achieved when the elastic term in formula (1) is not larger than $3 \%$ of the main frequency. For a Beryl-Bronze wire of diameter $90 \mu \mathrm{m}$ and tension of $100-250 \mathrm{MPa}$ this condition is satisfied when the wire length is in the range of $30-50 \mathrm{~mm}$. Such lengths provide a large enough operational aperture about $12 \mathrm{~mm}$, while approximately $\frac{2}{3}$ of the wire is covered by the permanent magnets necessary for the oscillations excitation.

Operational range of the $V W S$ : In the following we estimate the thermal fluxes to the Beryl-Bronze wire of length $l=36 \mathrm{~mm}$ and radius $r=0.045 \mathrm{~mm}$ at its different positions in the beam halo. Other physical parameters are

coefficient of thermal conductivity: $\quad \lambda=0.17 \mathrm{~W} / \mathrm{K} / \mathrm{mm}$ coefficient of thermal expansion: $\alpha_{\mathrm{S}}=1.9 \times 10^{-5} \mathrm{~K}^{-1}$.

The ionization loss of protons of energy $15 \mathrm{GeV}$ in Bronze are $\mathrm{d} E / \mathrm{d} s=1.3 \mathrm{MeV} / \mathrm{mm}$ and corresponds to an average heating quantity of $q_{1}=27.57 \mathrm{keV}$ from one particle hitting the wire. Table 2 presents the results of the estimations of $I_{\mathrm{S}}, Q_{\mathrm{S}}, T_{\text {mean }}$ at different positions $x$ of the wire with respect to beam center for protons of a current of $I_{0}=10 \mathrm{~mA}$ and $\sigma_{x}=6 \mathrm{~mm}$. The wire is stretched along the vertical axis $z$. A start position of $40 \mathrm{~mm}$ from the center of the vacuum chamber ("park position") was chosen to provide a minimal influence of the scanner on the beam dynamics in PETRA's common operation modes.

Note that the area at more than $2.5 \sigma$ is often called beam halo or "tails" [13]. Since we were interested in the beam tails of PETRA we chose $17 \mathrm{~mm}$ as the minimal distance of the scanner from beam center. A seen from Table 2 in this position the wire overheating is about $70^{\circ} \mathrm{C}$. This parameter defines the necessary initial stress of the wire. The stress of $2.8 \times 10^{8} \mathrm{~Pa}$ provides an operational range over $90^{\circ} \mathrm{C}$ (ratio $E / \sigma$ in formula (1) is equal to 464 ). At this stress the oscillation frequency of the first harmonics is about $2450 \mathrm{~Hz}$. Since the magnetic system partly covers the wire we choose the scheme of second harmonics excitation, thus leaving the central part of the wire free for interaction with the beam. The second harmonics shift $\Delta F$ calculated by the formula (5) at different positions of the wire is also presented in Table 2. It is seen that at wire position $17 \mathrm{~mm}$ from the vacuum chamber center the frequency shift is about $1500 \mathrm{~Hz}$. We modeled such frequency shifts in experiments, where the wire heating was done by a transmission of a direct current through the wire. These tests showed that multiple shifts of the frequency by this value do not lead to hysteresis and drifts of the frequency.

Normally the frequency noise was less than $0.01 \mathrm{~Hz}$ at every-second measurement. As seen from Table 2 this value corresponds to the wire position from the beam center of almost $35 \mathrm{~mm}$ and a wire overheating of $0.0005^{\circ} \mathrm{C}$ only.

To summarize, we choose the wire position range of $17-35 \mathrm{~mm}$ ( $\equiv 2.8$ to $5.8 \sigma)$ where the beam heat power deposited on the wire changes from $2.7 \times 10^{-2}$ to $8.9 \times 10^{-8} \mathrm{~W}$.

\section{Vibrating wire scanners, experimental results}

The main view of the VWS developed for PETRA is presented in Fig. 4. Special construction of the clips (1) provides stable fastening of the wire ends during the long time experiments. The support of the VWS was made from quartz (2) with low coefficient of thermal expansion. Since the scanner was developed for profiling the halo of a charged particle beam, special magnet poles were developed to diminish the magnetic field dissipation ((3) and (5)). The working area of the VWS lies in the central part of the vibrating wire (6). As mentioned above in this case the second harmonic of natural oscillations is exited.

Table 2

Some typical parameters of Beryl-Bronze wire heating process by $10 \mathrm{~mA}$ proton beam

\begin{tabular}{llllll}
\hline$x(\mathrm{~mm})$ & $I_{\mathrm{S}}(x)(\mathrm{A})$ & $I_{\mathrm{S}}(x) / I_{\mathrm{S}}(0)$ & $Q_{\mathrm{S}}(\mathrm{W})$ & $T_{\text {mean }}(\mathrm{K})$ & $3.45 \mathrm{E}+02$ \\
\hline 13 & $5.066 \mathrm{E}-06$ & $1.019 \mathrm{E}-01$ & $1.395 \mathrm{E}-01$ & $6.82 \mathrm{E}+01$ & $1.473 \mathrm{E}+03$ \\
17 & $1.001 \mathrm{E}-06$ & $2.014 \mathrm{E}-02$ & $2.760 \mathrm{E}-02$ & $1.52 \mathrm{E}+01$ & $3.287 \mathrm{E}+02$ \\
20 & $2.238 \mathrm{E}-07$ & $4.494 \mathrm{E}-03$ & $6.160 \mathrm{E}-03$ & $1.28 \mathrm{E}-01$ & $1.572 \mathrm{E}+01$ \\
25 & $1.069 \mathrm{E}-08$ & $2.149 \mathrm{E}-04$ & $2.945 \mathrm{E}-04$ & $2.19 \mathrm{E}-04$ & $4.825 \mathrm{E}-01$ \\
30 & $2.592 \mathrm{E}-10$ & $5.229 \mathrm{E}-06$ & $8.89 \mathrm{E}-06$ & $1.38 \mathrm{E}-06$ & $2.984 \mathrm{E}-05$ \\
35 & $3.214 \mathrm{E}-12$ & $6.475 \mathrm{E}-08$ & $5.587 \mathrm{E}-10$ & 08 & \\
40 & $2.020 \mathrm{E}-14$ & $4.079 \mathrm{E}-10$ &
\end{tabular}

The wire with a diameter of $90 \mu \mathrm{m}$ and length of $36 \mathrm{~mm}$ is placed at the different distances $x$ from the beam center. The heat power $Q_{\mathrm{S}}$ and the equilibrium mean temperature $T_{\text {mean }}$ of the wire correspond to the shift of the oscillations frequency relative to the initial frequency $4850 \mathrm{~Hz}$. 
In this development phase we used only one vibrating wire. Usage of few wires will widen the VWS application area [14].

\subsection{Wire oscillation excitation}

Wire natural oscillations arise as a result of the interaction between the oscillating current through the wire and magnetic field of the samarium-cobalt permanent magnets. The magnet provides field strength on the order of $10 \mathrm{kGs}$ in the gap. When the oscillating current passes through the wire the Lorentz-force shifts the wire in the transverse

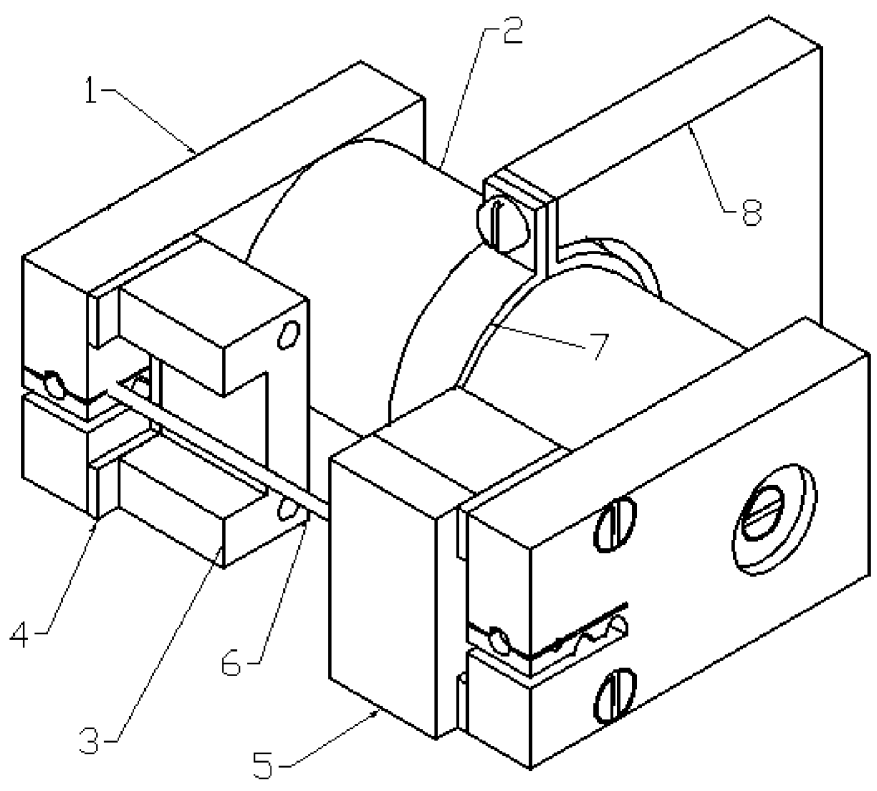

Fig. 4. Vibrating wire scanner main view with parts description: 1 - one of the clips (is fastened on the VWS support butt-end), 2 - the sensor support made of material with low coefficient of thermal expansion, 3, 5-magnet poles system, 4 - dielectric plates, 6-vibrating wire, 7, 8-VWS fasteners. direction by a value proportional to the magnitude of the magnetic field and the peak current. The value of the induced voltage is proportional to the time derivative of the current, which from the electrical viewpoint is equivalent to an inductance. So the electrical equivalent of the wire can be described as a series of a resistor and an inductance (see e.g. Ref. [15]). Thus, excitation of mechanical oscillations in the wire is possible because the wire acts like a tuned circuit when placed in an amplifier feedback arrangement.

When the wire is connected to an amplifier circuit a small amount of energy is fed back to the wire, which causes it to vibrate. This is similar to the excitation of electromechanical oscillations in quartz resonators (see e.g. Ref. [16]).

The wire is connected in a positive feedback circuit, which is the lower arm of the divider. The output of the divider is connected to the noninverting input of the operating amplifier to support the oscillations. To keep the amplitude of oscillations within a definite level an amplitude comparator on the base of operational amplifier is provided and connected to the negative feedback circuit.

The frequency measurement was done by counting the short impulses of the quartz generator inside the few periods of measured oscillations.

\subsection{Electron beams}

The first scanning experiments on a charged beam were done on an electron beam at the Injector of Yerevan Synchrotron with an average current of about $10 \mathrm{nA}$ (after collimation) and an electron energy of $50 \mathrm{MeV}$. The VWS used was approximately the same as described above.

Fig. 5 represents the result of the reconstruction of the beam profile for the first half-distance scanning (at the vertical axis the beam current density in horizontal direction is presented). The solid line represents the profile

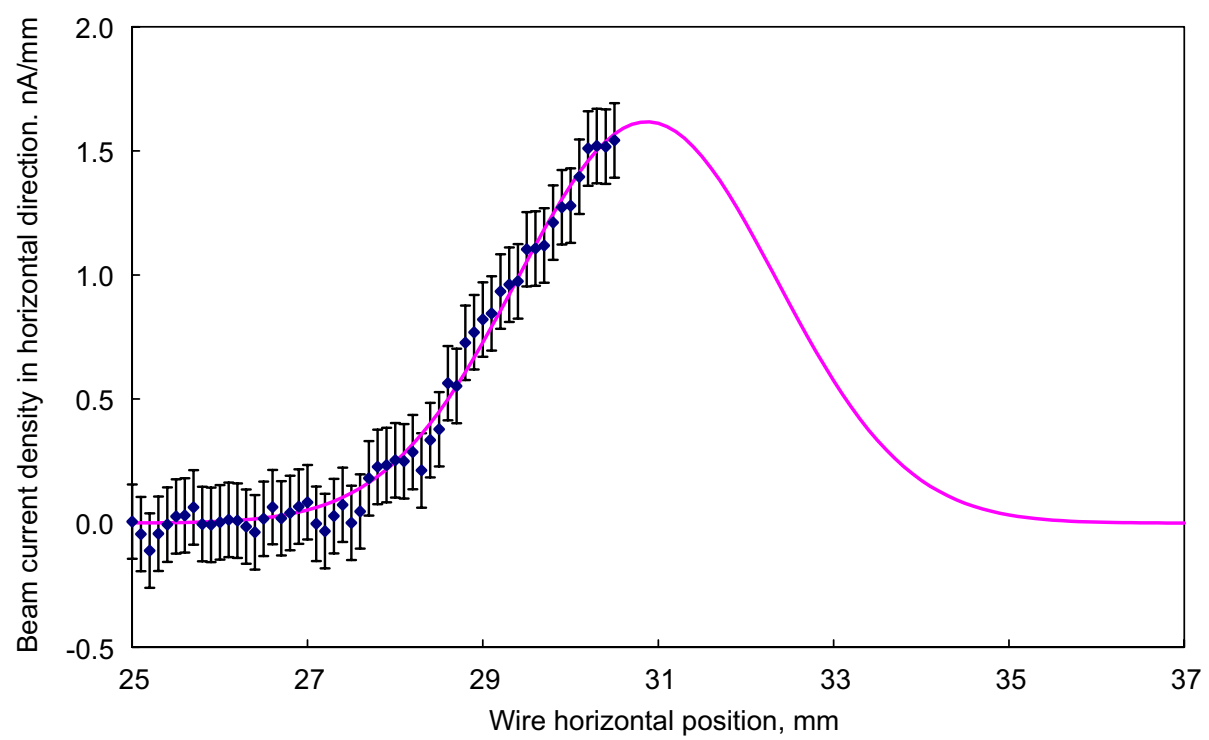

Fig. 5. Reconstruction of the horizontal profile of a $20 \mathrm{MeV}$ electron beam with a current of $10 \mathrm{nA}$ scanned with the VWS. At the vertical axis the beam current density in horizontal direction is presented. 
of the beam approximated by the mean square method of a Gaussian function with a standard deviation of $\sigma_{x}=$ $1.48 \mathrm{~mm}$ and a beam position at $30.87 \mathrm{~mm}$. The overall current of the beam was measured to be $I_{0}=10 \mathrm{nA}$. The scan feed mechanism has some limitation so we have done scanning up to beam center and back. A more detailed description of the experimental results can also be found in Refs. [17-20].

\subsection{Ion beams}

The VWS was also tested on an ion beam of energomass-analyzer EMAL-2 [20,21]. The Iron ion beam current of about $1 \mathrm{nA}$ and an energy of $20 \mathrm{keV}$ was obtained. The VWS was placed at the central position of the beam (see Fig. 6).

The ion beam was locked by a diaphragm and only in a period between 15 and $30 \mathrm{~s}$ the ion beam hit the wire. Approximately $16 \mathrm{pA}$ of beam current interacted with the wire and a frequency decrement of about $0.15 \mathrm{~Hz}$ was measured.

The interest to continue ion beam profiling by the VWS is stimulated by the fact that the vibrating wire scanning method is based on thermal action of the beam and accumulates effect from both charged and neutral particles. So by this method the effect of electrical neutralization of the ion beam by electron clouds can be overcome.

\subsection{Proton beams}

A series of experiments with the VWS were done on proton beam of the accelerator PETRA at DESY. The place for the VWS location was chosen in the so-called "proton by-pass" to avoid any influences or distractions from higher order mode losses created by short electron bunches which are accelerated in the same machine but did not enter the "by-pass". For the scanning experiments the beam was specially prepared for our purposes and consisted of 10 bunches with an initial mean current of about $15 \mathrm{~mA}$ and an energy of $15 \mathrm{GeV}$. The transversal beam sizes were: $\sigma_{x}=6 \mathrm{~mm}, \sigma_{z}=5 \mathrm{~mm}$. A system of two scintillator-photomultiplier pickups (PM1 and PM2) were installed additionally to measure particles scattered on the wire. The park position of the wire is located at the $40 \mathrm{~mm}$ $\left(6.7 \sigma_{x}\right)$ from the center of the vacuum chamber on the outside of the accelerator ring. The scanner is driven by a stepping motor toward to the vacuum chamber center. A system of adjustable beam bumps allows to control the position of the beam inside the vacuum chamber at the scanner location.

Since in some operation modes of the PETRA accelerator the proton beam current exceeds $100 \mathrm{~mA}$, even in park position the VWS actively reacted to changes of beam parameters by slow changes of environment temperature.

Fig. 7 represents the typical picture of the scanner frequency change in park position during $30 \mathrm{~h}$. The proton and electron beams currents in PETRA are also presented. The temperature change in the chamber without beam can be estimated from the data to be about $7^{\circ} \mathrm{C}$. In the presence of proton beam current the frequency behavior depends on current intensity. At currents less than $50 \mathrm{~mA}$ the wire frequency changes are proportional to the beam current while at higher currents probably the electromagnetic disturbances also have influence on the frequency/ temperature by absorbing some modes.

The event at 20.05.2004 15:00 when the frequency rapidly decreases might be explained by thermal effects due to some high current electron beam operation.

In a stationary position the frequency smoothly drifts, as a rule, caused by a change of beam current and environmental conditions. The root-mean-square deviation of the frequency is about $0.01 \mathrm{~Hz}$ in the VWS stable

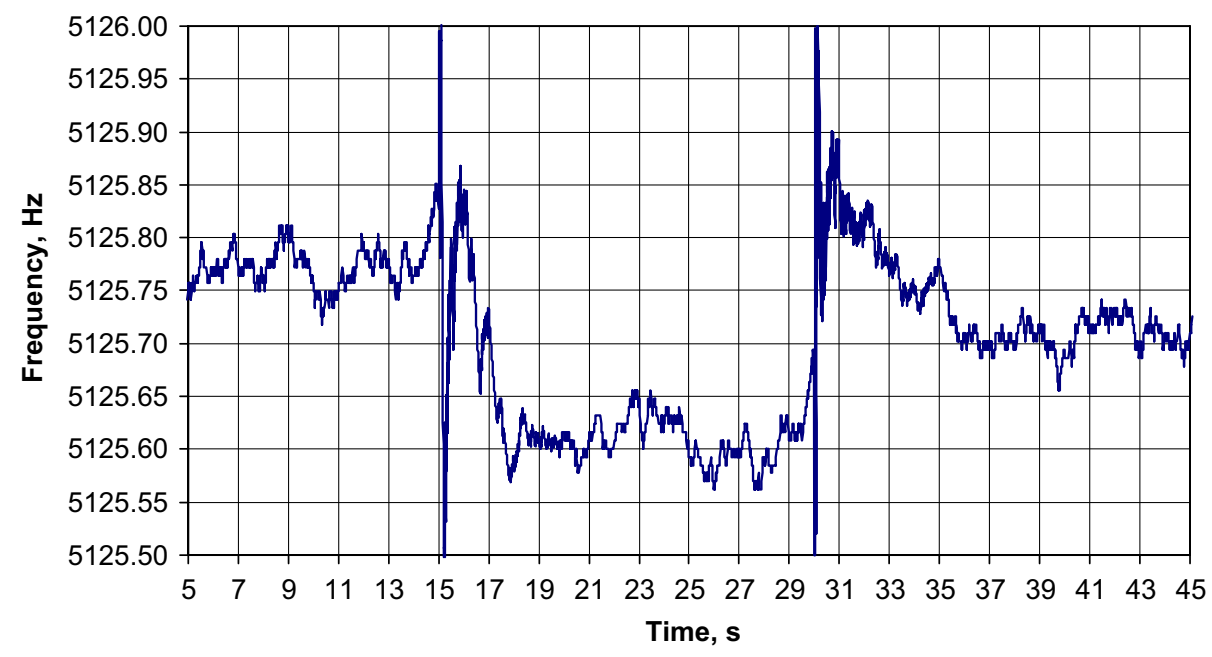

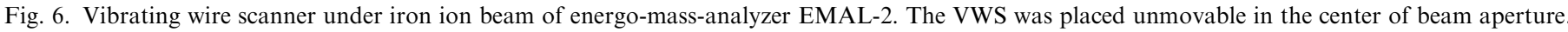

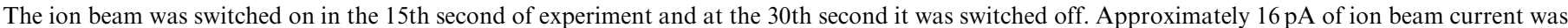
deposited onto the wire. 


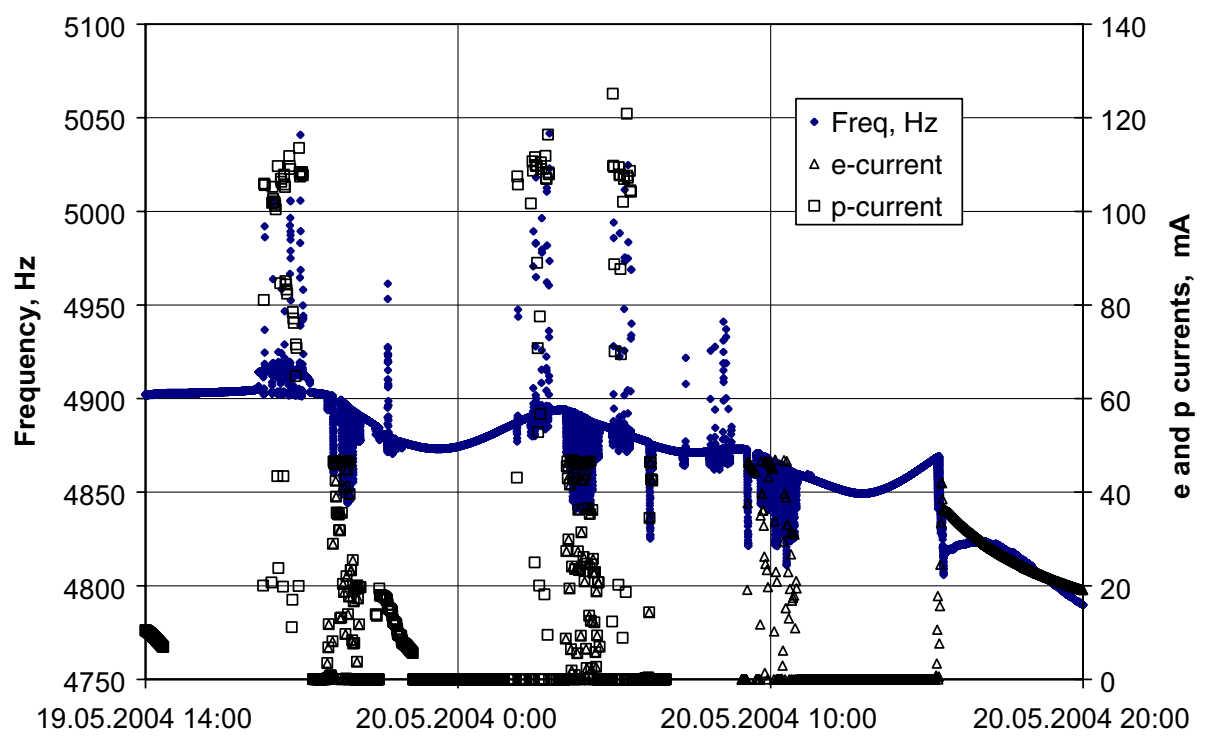

Fig. 7. Dependence of VWS frequency in park position $-40 \mathrm{~mm}$ from the vacuum chamber center in proton by-pass. Available values of proton (squares) and electron (triangles) beam currents are also presented. In absent of the beam the frequency changes smoothly according to the environmental temperature with sensitivity about $20 \mathrm{~Hz} / \mathrm{K}$.

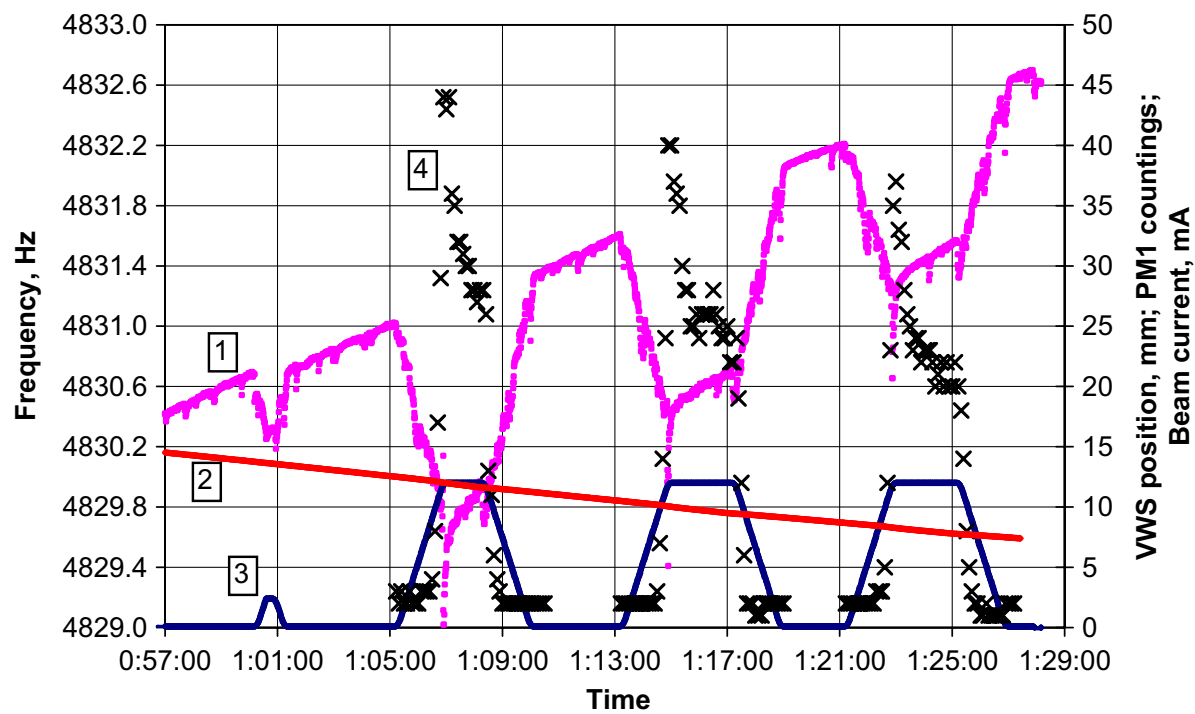

Fig. 8. Some shallow scans of $15 \mathrm{GeV}$ proton beam by vibrating wire scanner. The VWS feed was started from park position ( $40 \mathrm{~mm}$ from vacuum chamber center) by a distance of $12 \mathrm{~mm}$ toward to the beam center (besides the first shallow scan by $2 \mathrm{~mm}$ distance). Curves indicate: $1-$ frequency signal from VWS, 2- beam current, 3-VWS distance from park position, 4-scattered particle counting pickup PM1 countings (denoted by crosses). During the experiment (about half an hour) the proton current decrease from 14 to $7.5 \mathrm{~mA}$.

position during approximately $10 \mathrm{~min}$ time interval. This time interval is large enough for full scanning. The corresponding change of the wire mean temperature is about $0.0005^{\circ} \mathrm{C}$.

The results of beam scanning with additional measurements of scattered particles by the scintillation counter PM1 are presented in more detail in Fig. 8. Fig. 8 shows the results of a scan of $12 \mathrm{~mm}$ from the park position together with the readings of PM1.

The beam shift from the center of vacuum chamber was: $z=3.1 \mathrm{~mm}, x=7.6 \mathrm{~mm}$. One shallow scan up to the distance of $2.5 \mathrm{~mm}$ from the park position and three consequent scans up to $12 \mathrm{~mm}$ were done. Fig. 8 shows that pickup PM1 showed signals only when approaching a distance of $10 \mathrm{~mm}$ from park position. The entire experiment lasts about $1.5 \mathrm{~h}$ and during this period the beam current monotonously decreased from 14.5 to $7.4 \mathrm{~mA}$. No additional beam current losses were observed as a result of the larger distance between the wire and the beam center. In accordance with the overall decrease of the current a monotonous increase of the frequency of the scanner in park position as well as at $12 \mathrm{~mm}$ occurs. We suppose that this effect is caused by a decrease in the vacuum chamber temperature. 
The results of the VWS at positions of $12 \mathrm{~mm}$ are accompanied with spikes in the frequency signal. Similar effects (more blurred) are observed in the data obtained from pickup PM1. These spikes may be caused by some beam halo scraping on the VWS support. Note that the $\frac{2}{3}$ part of the operating oscillating wire is covered by the magnetic system of the scanner (see Fig. 4). Actually the magnetic poles are close to the active vibrating wire, therefore a fairly great number of particles can scatter on it.

Another example is shown in Fig. 9. Again the signal from the VWS sensor changes from the beginning of the movement, while the signals from scintillators start to increase only at distances of $13 \mathrm{~mm}$ from the VWS park position.

The whole scan feed was $20 \mathrm{~mm}$. In this experiment the proton beam was shifted towards the scanner park position by a distance of $4 \mathrm{~mm}$ by means of a local beam bump. During the experiment $(20 \mathrm{~min})$ the proton beam current decreased from 12 to $9.5 \mathrm{~mA}$. At the same time the frequency decreased by $1 \mathrm{~Hz}$. In stable environmental conditions without beams such behavior was not observed. This effect can be explained by change in environmental temperature and can be taken into account at beam transversal profile reconstruction. Another possibility is to use more than one wire in different positions relative to the beam.

As seen from Figs. 8 and 9, the signal from the VWS appears at feed positions where the signal from the scintillators is absent. Some contribution in wire heating might occur from the influence of electromagnetic higher order modes accompanying the proton beam. These electromagnetic components might are able to heat the wire by absorbing some modes (see Ref. [22]). Clarification of this problem and corresponding modifications of the VWS require additional efforts.
Despite this effect, the signals from PM1 and PM2 are strongly correlated with the frequency signal from the VWS (see Fig. 10). Some hysteresis occurs which is related to the different direction of the VWS movement.

The largest shift of the wire oscillation frequency due to heating was measured to be about $150 \mathrm{~Hz}$, at a horizontal distance between the wire and beam center slightly less than $20 \mathrm{~mm}$. This value is about a factor 2 less than the calculated value given in Table 2. Two effects might contribute to the uncertainty: (1) the uncertainty of the absolute beam position relative to vacuum chamber of $\pm 1.5 \mathrm{~mm}$; (2) non-Gaussian beam profiles.

The role of electromagnetic background also needs further investigation. The heating of the wire by electromagnetic modes of the proton beam seems to be not completely negligible, even at very far distances. We expect, that this effect will be much more dominant in the case of the much shorter bunches of an electron beam, but much less in case of DC beams. To determine this effect additional experiments are required.

\section{Conclusion}

For the suggested application in accelerator beams diagnostics it is very important that the VWS can operate in hard conditions and that the sensor frequency signal can be transmitted over long cable with no loss or degradation. The VWS scanner is a compact unit while typical existing wire scanners needed an additional unit for secondary radiation measurement normally placed at few meters downstream from the target. The thermal character of the interaction between the measured beam and the wire requires some time to establish thermal equilibrium and limits the scan speed. However, the method's very much higher sensitivity compared to available scanners allows the

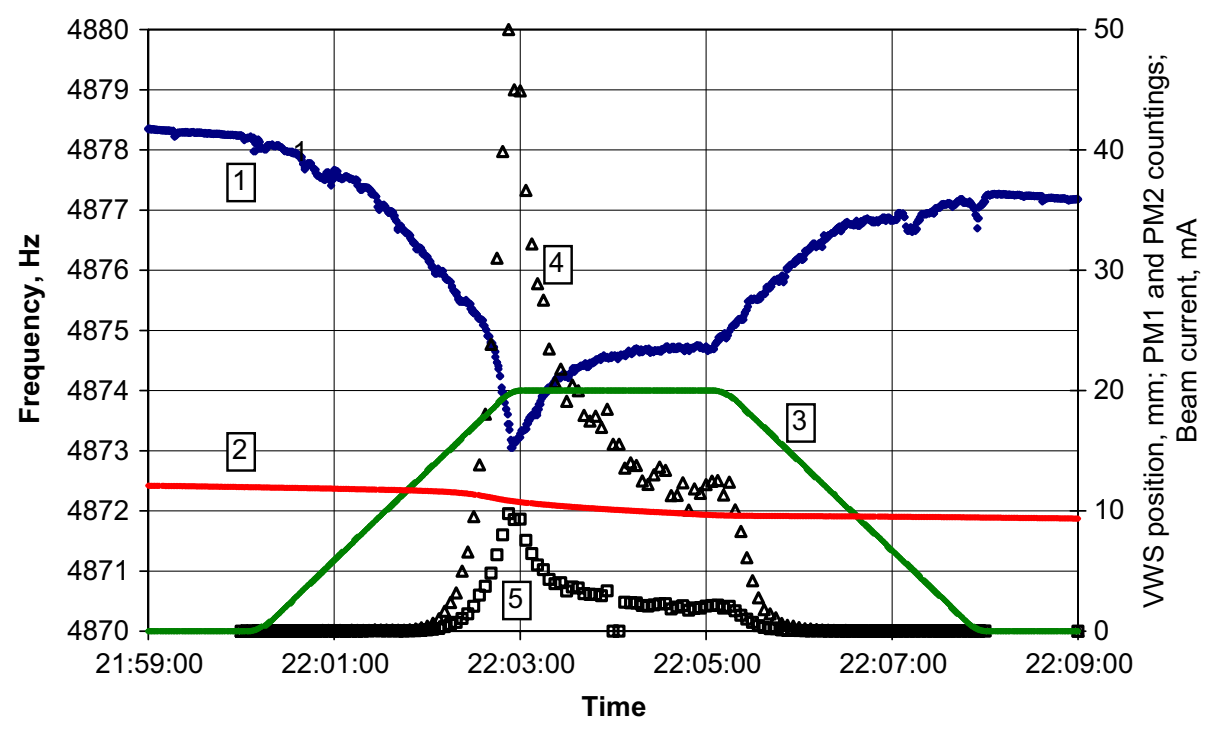

Fig. 9. Scan of the proton beam using the VWS: 1-frequency of the VWS, 2-beam current, 3-VWS feed from the park position toward to the beam center, 4 and 5-signals from scintillators PM2 (triangles), PM1 (squares). During experiment (20 min) the proton beam current decreased from 12 to $9.5 \mathrm{~mA}$. 


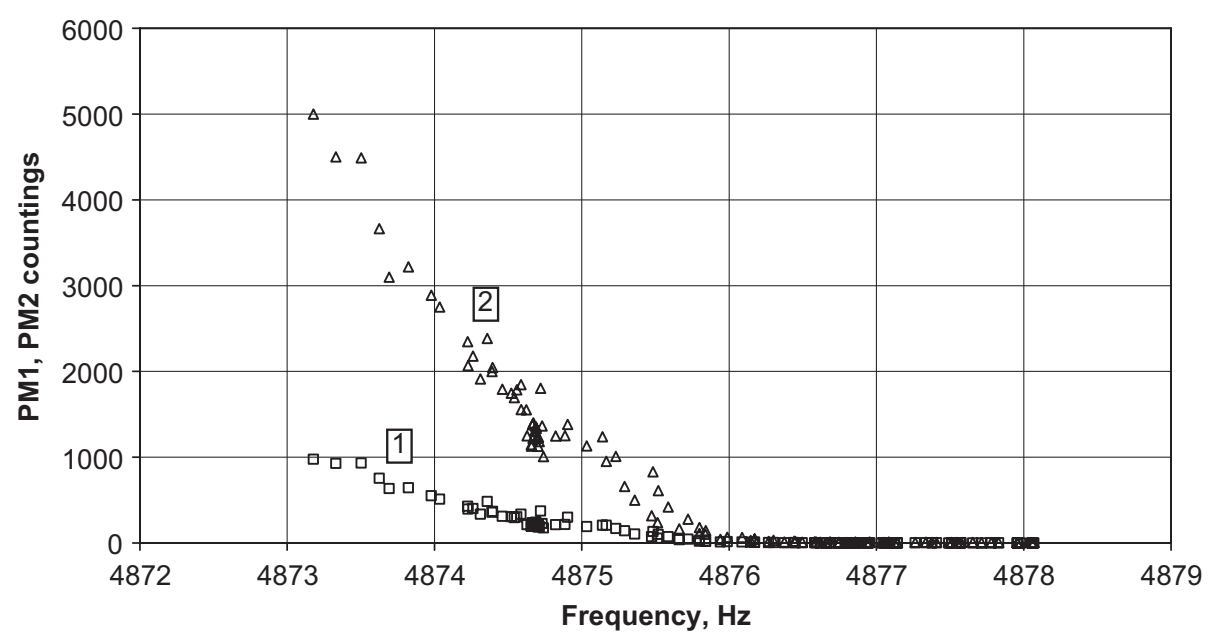

Fig. 10. Comparison of signals from the VWS with PM1 (curve 1, squares) and PM2 (curve 2, triangles) during the proton beam scan. The responses from PM1 and PM2 arise only at point when frequency signal decreases from 4878 to $4876 \mathrm{~Hz}$ that corresponds to the feed more than $10 \mathrm{~mm}$ from park position.

beams profiling at much greater distances from the beam center (halo) and at very low current beams ( $\ll 1 \mathrm{nA})$. E.g. experiments with the VWS proton beams of PETRA show that the system is sensitive to beam current changes at distance of $6.6 \sigma$ (here $40 \mathrm{~mm}$ ) from the beam center. The scanning shows a strong correlation between scanner position and frequency signal. The accuracy of the frequency measurements achieved is $\pm 0.01 \mathrm{~Hz}$ which corresponds to wire temperature measurement accuracy less than $\pm 0.001{ }^{\circ} \mathrm{C}$. Comparison of the signal from the VWS with a scintillating counter system registering scattered particles/radiation showed that the signal from the VWS appears at least $10 \mathrm{~mm}$ farther from the beam center than mentioned scintillators. Except this effect the signals are very well correlated.

At very large distances from the beam the heating of the wire due to higher order mode coupling can disturb the signal. Some more development is necessary to get rid of this effect. Very promising will be the development of sensors on basis of dielectric strings, including new type of oscillation excitation and data acquisition. The area of VWS applications can be enlarged, including profiling and positioning of photon beams from synchrotron light sources and laser beams [23]. In this case the electromagnetic coupling does not exist.

The data obtained show that the VWS can be used for very sensitive beam diagnostics in accelerators as a cheap, precise, reliable and robust sensor with wide range of applications.

The VWS can be used also for laser beam and for ion beam profiling with some small modifications [24].

\section{Acknowledgments}

The authors are grateful to the PETRA stuff for their friendly help during the experiments on PETRA.

\section{References}

[1] P. Elmfors, A. Fasso, M. Huhtinen, M. Lindroos, J. Olsfors, U. Raich, Nucl. Instr. and Meth., Phys. Res. A 396 (1-2) (1997) 13.

[2] S. Striganov, G. Schmidt, K. Wittenburg, Estimation of the signal from the wire scanner in the TTF /desyntwww.desy.de/mdi/ downloads/WIRE_TTF.pdf).

[3] H. Hayano, Wire scanners for small emittance beam measurement in ATF, Proceedings of XX International Linac Conference, 21-25 August 2000, Monterey, CA, USA, pp. 146-148.

[4] V. Agoritsas, E. Falk, F. Hoekemeijer, J. Olsfors, Ch. Steinbach, The fast wire scanner of the CERN PS, CERN-PS-95-06-BD-OP.

[5] S.G. Arutunian, N.M. Dobrovolski, M.R. Mailian, I.G. Sinenko, I.E. Vasiniuk, Vibrating wire scanner for beam profile monitoring, Proceedings of the Particle Accelerator Conference, March 29-April 2, 1999, New York City, pp. 2105-2107.

[6] S.G. Arutunian, N.M. Dobrovolski, M.R. Mailian, I.G. Sinenko, I.E. Vasiniuk, Phys. Rev. Spec. Top. Accel. Beams 2 (1999) 122801.

[7] S.G. Arutunian, M. Werner, K. Wittenburg, Beam tail measurements by wire scanners at DESY, ICFA Advanced Beam Dynamic Workshop: Beam HALO Dynamics, Diagnostics, and Collimation (HALO'03) (in conjunction with third workshop on beam-beam interaction), May 19-23, 2003, Gurney's Inn, Montauk, NY, USA.

[8] T. Simmonds, In today's ever growing and diversifying market place vibrating wire based measurement systems are, and will continue to be, highly significant, Monosys Guide of Monitoring, 2000, Q-2, pp. 14-16.

[9] F. Ayazi, K. Najafi, J. Micromech. Syst. 10 (2) (2001) 169.

[10] A.M. Kosevich, E.M. Lifshitz, L.D. Landau, L.P. Pitaevskii, Theory of Elasticity, Butterworth-Heinemann, London, 1986.

[11] I.S. Grigor'ev, E.Z. Meilikhov, Physical Constants, Moscow, EnergoAtomIzdat, 1991.

[12] D.T. Attwood, B.L. Henke, Low energy X-ray diagnostics, AIP Conference Proceedings, vol. 75, American Institute of Physics, New York, 1981.

[13] L. Cremaldi, I. Ostrovskii, /www.hep.uiuc.edu/LCRD/EOI_docs/ BEAM_HALO_MONITOR.pdf $\rangle$.

[14] S.G. Arutunian, N.M. Dobrovolski, M.R. Mailian, V.A. Oganessian, I.E. Vasiniuk, Vibrating wires fence as a negligibly destructive beam profile and beam position monitor, Proceedings of the NATO Advanced Research Workshop on Electron-Photon Interaction in Dense Media, 25-29 June 2001, Nor Amberd, Armenia, NATO Science Series; II Mathematics, Physics and Chemistry, vol. 49, pp. 303-308. 
[15] Ju.F. Elcev, V.M. Zakosarenko, V.I. Tsebro, String magnetometer, Transition processes in superconducting magnetic systems, Trudy FIAN, vol. 150, Moscow, pp. 141-147.

[16] Fundamentals of Quartz Oscillators, Application Note 200-2, Hewlett Packard, Electronic Counters Series.

[17] S.G. Arutunian, A.E. Avetisyan, N.M. Dobrovolski, M.R. Mailian, I.E. Vasiniuk, K. Wittenburg, R. Reetz, Problems of installation of vibrating wire scanners into accelerator vacuum chamber, Proceedings of Eighth European Particle Accelerator Conference, 3-7 June 2002, Paris, France, pp. 1837-1839.

[18] S.G. Arutunian, K.G. Bakshetyan, N.M. Dobrovolski, M.R. Mailian, A.E. Soghoyan, I.E. Vasiniuk, First experimental results and improvements on profile measurements with the vibrating wire scanner, Proceedings of DIPAC'2003, 5-7 May, Mainz, Germany 2003, pp. 141-143.

[19] S.G. Arutunian, N.M. Dobrovolski, M.R. Mailian, I.E. Vasiniuk, Phys. Rev. Spec. Top. Accel. Beams 6 (2003) 042801.

[20] S.G. Arutunian, K.G. Bakshetyan, N.M. Dobrovolski, M.R. Mayilyan, V.A. Oganessian, A.E. Soghoyan, I.E. Vasiniuk, K. Wittenburg, Vibrating wire scanner parameters optimization, Proceedings of Nineth
European Particle Accelerator Conference, 5-9 July 2004, Lucerne, Switzerland, pp. 2457-2459.

[21] M.A. Aginian, S.G. Arutunian, V.A. Hovhannisyan, M.R. Mailian, K. Wittenburg, Vibrating wire scanner/monitor for photon beams with wide range of spectrum and intensity, NATO Advanced Research Workshop "Advanced Photon Sources and their Application," Nor Amberd, Armenia, August 29-September 02, 2004.

[22] F. Caspers, B. Dehning, E. Jensen, J. Koopman, J.F. Malo, F. Roncarolo, Cavity mode related wire breaking of the SPS wire scanners and loss measurements of wire materials, Proceedings of DIPAC'2003, 5-7 May, Mainz, Germany, 2003, pp. 119-121.

[23] S.G. Arutunian, N.M. Dobrovolski, M.R. Mailian, V.A. Oganessian, I.E. Vasiniuk, Nonselective receiver of laser radiation on the basis of vibrating wire, Proceedings of Conference Laser 2000, November 2000, Ashtarak, Armenia.

[24] M.A. Aginian, S.G. Arutunian, M.R. Mailian, Vibration wire monitor for photon beams diagnostics: preliminary tests on laser beams, NATO Advanced Research Workshop, CANDLE, 17-21 July 2006, Yerevan, Armenia, Brilliant Light Facilities and Research in Life and Material Sciences. 